\title{
Ethos platonico, poiesis aristotelica e metafisica tridentina nelle tragedie degli anni 1630
}

\section{Vincenzo Reina Li Crapi}

\section{(2) OpenEdition}

1 Journals

\section{Edizione digitale}

URL: https://journals.openedition.org/cher/1990

DOI: $10.4000 /$ cher. 1990

ISSN: 2803-5992

\section{Editore}

Presses universitaires de Strasbourg

\section{Edizione cartacea}

Data di pubblicazione: 29 mai 2018

Paginazione: 21-42

ISBN: 979-10-344-0019-5

ISSN: 1968-035X

\section{Notizia bibliografica digitale}

Vincenzo Reina Li Crapi, «Ethos platonico, poiesis aristotelica e metafisica tridentina nelle tragedie degli anni 1630», reCHERches [Online], 20 | 2018, online dal 01 décembre 2021, consultato il 02 décembre 2021. URL: http://journals.openedition.org/cher/1990 ; DOI: https://doi.org/10.4000/cher. 1990

\section{(c) (i) (3) (-)}

Ce(tte) œuvre est mise à disposition selon les termes de la Licence Creative Commons Attribution -

Pas d'Utilisation Commerciale - Partage dans les Mêmes Conditions 4.0 International. 


\title{
Ethos platonico, poiesis aristotelica e metafisica tridentina nelle tragedie degli anni 1630
}

\author{
Vincenzo Reina Li CRAPI ${ }^{1}$
}

$\mathrm{L}$ a decade del 1630 segna una svolta nella storia letteraria francese. Nel 1628 «la tragédie est morte» (Forestier 1993: 87); a partire da questo momento inizia tuttavia un dibattito che culmina con l'imposizione di un gusto «classico moderno» e di norme prestabilite per ogni pièce che aspiri ad avere uno statuto

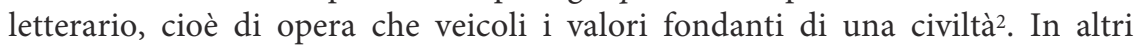
termini d'ora in poi i drammaturghi saranno obbligati a rispettare delle regole che rispondono a delle esigenze sia funzionali che etiche, legate alla fruizione dell'opera. Le fasi di inventio e dispositio permettono di cogliere la maniera di riscrivere una storia al fine di poterla rappresentare, adattandola al gusto del pubblico - potere politico; esse si fanno così portatrici di senso ${ }^{3}$. Il procedimento di "genetica teatrale» di Corneille ne è un esempio lampante, dal momento che lo scioglimento si configura come «l'élément fondamental» di una tragedia, e «en même temps le sujet de l'œuvre» (Forestier 2004: 15).

Per i drammaturghi coevi la riscrittura di una storia è subordinata principalmente al rispetto dell'unità di tempo e del verosimile. Invero, dietro queste regole si cela il bisogno, cosciente o meno, di veicolare il modo di sentirsi e di

1 Lecteur à l'Université de Corse.

2 Rinviamo ai lavori di A. Duprat su J. Chapelain.

3 Non è soltanto «la tragédie cornélienne [qui] produit le sens par la forme» (Forestier 2004: 358). Parlando della scrittura teatrale al tempo di Richelieu, G. Couton (1986: 22) ha segnalato che «on distingue deux temps dans l'élaboration. Le premier temps est celui de "l'invention" ou du "dessein", c'est-à-dire la recherche du sujet, suivi de la "disposition", c'est-à-dire de l'ajustement des incidents, de la construction de la pièce. Le deuxième temps, est celui de l'écriture. Les écrivains de théâtre du $\mathrm{XVII}^{\mathrm{e}}$ siècle considèrent que ce deuxième temps est la partie facile du travail». Tale idea è confermata dai numerosi reimpieghi di versi (Louvat-Molozay 2008). 
agire dell'uomo nel tempo ${ }^{4}$. Di seguito, dopo aver fatto un breve cenno al dibattito teoretico, ci proponiamo di svelare quali siano queste concezioni, attraverso appunto il lavoro di riscrittura compiuto dal drammaturgo. Soffermandoci in particolare sull'Horace e sulla Mariane, tenteremo di dimostrare come dietro la riscrittura di storie ci sia non solo il bisogno di obbligare «l'œil surpris à se tromper lui-même pour son profit» (Chapelain 2007: 225), ma anche quello di esprimere, oltre all'ideale di magnanimità (Fumaroli 1996), la libertà della volontà affermata da Cartesio e ancor prima dal concilio tridentino (dissentire possit, si velit); nonché l'indispensabile subordinazione dell'azione dell'individuo - e della letteratura - al bene della sua comunità, così com'è affermato nella Repubblica di Platone.

Da un lato verrà posto l'accento sul fatto che una storia, dal momento in cui viene riscritta per essere messa in scena, perde l'originario messaggio per acquisire una valenza ideologica nuova: rivelatrice in questo senso è la Mariane, che sconvolge il valore di storia esemplare contenuta nella Cour sainte del P. Caussin. La scelta del genere della tragedia, peraltro, permette a Tristan l'Hermite di formulare una visione del mondo molto più problematica (Mazouer 2015: 423), che tenga anche conto dei dibattiti coevi sulla libertà umana, mettendone in luce le relative aporie.

Nel caso di Corneille, che torna a scrivere per il teatro dopo la condanna del Cid, la scelta del soggetto da trattare è già in parte rivelatrice dell'intenzionalità dell'opera: accettazione di una volontà politica che prende i tratti di una potestas absoluta (Merlin 1994), e che utilizza la letteratura per imporre la propria visione della società. Proprio nell'ottica dei rapporti tra cittadino e Stato, la scelta di portare sulla scena l'exploit di Orazio - che potrebbe leggersi anche come riscrittura di un tipo di azione già evocata come modello nel IV libro della Repubblica - è abbastanza significativa. Tale rimando significherebbe, in altri termini, abiurare la visione che si disegnava in filigrana nel Cid, per veicolare un tipo di ethos, tanto poetico quanto civile, simile a quello proposto dal modello platonico.

\section{Eredità italiana e aristotelismo in Francia: Chapelain e Mairet}

La polemica sul rispetto dei precetti aristotelici e in particolare sull'unità di tempo, iniziata nel 1628 con le Préfaces di Hardy e Ogier (Dotoli 1997: 18), è essenzialmente chiusa qualche anno dopo: nel 1632 le due fazioni principali sono ormai costituite, le principali argomentazioni sono già state esposte, e Chapelain, riformulando l'idea di piacere e di gusto del pubblico, priva gli avversari del fondamento dei propri argomenti (Forestier 1993: 105). Ma «l'avancée décisive»

4 Come ha intuito P. Ricœur (1983: 67) «le temps devient temps humain dans la mesure où il est articulé sur un mode narratif [...] imiter ou représenter l'action, c'est d'abord pré-comprendre ce qu'il en est de l'agir humain: de sa sémantique, de sa symbolique, de sa temporalité. [...] Pré-compréhension commune au poète et à son lecteur». 
di Chapelain non sarebbe stata tale se non avesse potuto beneficiare del successo della drammaturgia di Mairet. Entrambi sono giustamente tra i protagonisti dei due poli - teorico il primo, l'altro piuttosto votato alla sperimentazione attraverso i quali vengono diffuse Oltralpe le interpretazioni della Poetica dei teorici italiani e le sperimentazioni teatrali che in Italia hanno avuto luogo nel corso del Cinquecento.

Chapelain, animatore di diversi circoli letterari tra i quali l'Hôtel di Rambouillet, introduce nel dibattito francese le idee sul ruolo della letteratura sviluppate dagli italiani (Blocker 1999). Punto focale della sua teorizzazione è la necessità per l'opera letteraria di "proposer à l'esprit, pour le purger de ses passions déréglées, les objets comme vrais et comme présents» (Chapelain 2007: 223), idea che ha potuto ritrovare nei Discorsi sull'arte poetica del Tasso. Tuttavia il ricorso alle interpretazioni dei teorici italiani (e non solo) per formulare e dare un fondamento autoriale alla propria idea di letteratura non sarebbe stato sufficiente a convincere i sostenitori di un genere come la tragi-comédie, non vincolata alle unità aristoteliche. Del resto l'esempio stesso della tragedia regolare italiana, divenuta «un terrain d'expérimentation pour la théorie d'Aristote», rafforza la posizione di coloro che antepongono il diletto all'utilità della pièce ${ }^{5}$, poichè «si cet exercice peut plaire aux doctes, il ne convient pas au public» (Zanin 2014: 126).

Sono le sperimentazioni di Mairet, iniziate nella brillante corte di Chantilly e culminate col successo della Sophonisbe, a provare che è possibile creare una pièce che rispetti le regole e sia allo stesso tempo fonte di diletto, legittimando così le teorie formulate da Chapelain sul piacere. Per quest'ultimo il vero piacere teatrale scaturisce da una rappresentazione che sia capace di trasmettere l'illusione della verità. Orbene, per ottenere tale risultato è indispensabile che la costruzione della pièce sia verosimile; cioè che non esista nessuna discrepanza fra «représentant» e «représentation» (Forestier 1990: 192):

Je pose donc pour fondement que l'imitation en tous poèmes doit être si parfaite qu'il ne paraisse aucune différence entre la chose imitée et celle qui imite (Chapelain 2007: 223).

In altri termini si tratta per i futuri drammaturghi di creare una favola che «ne copie pas les choses, mais [qui] recrée le rapport de proportionnalité aux choses que nous avons dans notre perception habituelle du réel» (Hénin 2003: 290). Il riferimento all'esperienza sensoriale sta dunque alla base di un concetto di verosimile che pone il drammaturgo di fronte a una doppia difficoltà: in primo luogo creare l'illusione teatrale, e soprattutto dopo mantenerla nel tempo. Per Chapelain infatti non si tratta più di stimolare l'immaginazione dello spettatore puntualmente (come accade nelle tradizioni drammatiche coeve irregolari), ma di conquistarla e tenerla costantemente nell'illusione: «du coup, l'effet

5 Come scrive Ogier nel 1628, «la poésie, et particulièrement celle qui est composée pour le théâtre, n'est faite que pour le plaisir et le divertissement» (Dotoli 1997: 184). 
poétique ne doit pas être soudain mais continué», come spiega A.Duprat (Chapelain 2007: 71). Anche Corneille, reticente ad accettare la nozione di verosimile, non rimane insensibile a tale necessità, mostrandosi preoccupato a tutti quegli inconvenienti che rischiano di disingannare il pubblico, come appunto i riferimenti temporali in manifesta contraddizione col tempo della rappresentazione (Corneille 1987: 171-172). Attuando in tal maniera Chapelain è sicuro che le emozioni della rappresentazione sono ben interiorizzate dallo spettatore, dato che esse passano per lo stesso canale delle impressioni che vengono dal mondo concreto (Duprat 2009: 323).

D'altro canto il rispetto dell'unità di tempo influenza le emozioni che veicola lo spettacolo. Sebbene la regola non sia indispensabile per produrre l'effetto tragico, tuttavia può accentuarne i caratteri o permettere di illustrare il significato tragico dell'opera. In effetti, se da un lato essa è motivata dal gusto letterario dell'epoca (la metonimia preferita alla metafora barocca, Hénin 2003: 268-273), dall'altro strutturando l'esperienza umana del presente fornisce agli spettatori un modo specifico di apprensione della temporalità, attraverso lo spettacolo di personaggi che agiscono sulla scena nel breve lasso di tempo che impone il rispetto della regola.

In questa prospettiva abbiamo messo in luce altrove tre aspetti del tragico legati all'unità di tempo nella Sophonisbe di Mairet: l'enfasi posta sull'ultimo giorno fatale; il senso di urgenza e l'impotenza dei protagonisti di fronte al rapido precipitarsi degli eventi; infine il senso di fragilità umana, accentuata dal carattere effimero delle condizioni intramondane. Tali aspetti sono i risultati dell'operazione di poiesis compiuta da Mairet in fase inventio e dispositio ${ }^{6}$, e permettono di apprezzare in che modo la rappresentazione di un' «action qui tend si fort à la fin», come suggerisce Gombauld (Dotoli 1997: 258), o che si apre «le plus près qu'il est possible de la catastrophe» (D’aubignac 2001: 190) influenzi il carattere tragico dell'opera.

\section{La Mariane e il problema delle libertà umane}

\section{Dalla storia esemplare all'universo tragico}

In generale la tragedia pretende offrire agli spettatori l'immagine dell'uomo nei suoi rapporti con il mondo. Nel corso del ' 500 questo mondo è diventato via via più problematico come testimonia l'opera di scrittori e pensatori coevi, scossi dal susseguirsi di crisi politiche, religiose, epistemologiche ecc. Si è passati dal «monde clos» e razionale del tomismo all' «univers infini» dell'immaginazione bruniana. Orbene, in questa epoca in cui la filosofia calls all in doubt, secondo

6 Non siamo quindi pienamente d'accordo con J.-M. Civardi (2008: 68) quando scrive che «l'originalité du dramaturge réside plutôt dans l'adaptation qu'il en fait [de l'épisode] et dans ses options dramaturgiques quant à l'expression de la passion et de la fatalité - dans la dispositio plus que dans l'inventio». 
la celebre formula di J. Donne, la tragedia sembra essere la forma letteraria più appropriata per rappresentare un universo ambiguo e ingannevole.

La tragedia degli anni 1630 aspira a mettere in rilievo le facoltà umane e la maniera di impiegarle adeguatamente per condursi nel mondo. In particolare La Mariane di Tristan L'Hermite permette di cogliere alcuni aspetti salienti della rappresentazione dell'uomo del XviI secolo e dell'evoluzione della tragedia. Per l'immenso successo ottenuto al Marais (con la magistrale interpretazione di Mondory nella primavera del 1636), come per la sua ricerca insistente di emotività «entre Sophonisbe et Le Cid, La Mariane occupe une place de premier plan» (Scherer 1986: 1323). In effetti, come avevano ben capito i più fini teorici del Cinquecento, la «lezione» della tragedia non può esaurirsi nella semplice declamazione di sentenze. Accanto al docere e delectare oraziano, Scaligero aveva indicato un altro obiettivo che dovrebbe essere proprio della tragedia, il movere (da ciò derivano il suggerimento di Tasso a «guadagnarsi» l'animo dello spettatore e l'insistenza di Chapelain nel combinare indissolubilmente piacere e istruzione): obiettivo che secondo i teorici non potrebbe essere raggiunto senza il rispetto delle regole.

La Mariane sembra molto sensibile alle speculazioni teoriche sul teatro. Già nella fase di inventio mostra tale accortezza: Tristan sceglie un avvenimento narrato nella Cour sainte del gesuita Caussin, le cui storie comportano «une chute, un moment où les destins se resserrent dans une accélération fatale» (Hénin 2007: 107). Inoltre per il fatto di aver accordato all'azione drammatica "quelques heures», Tristan rende "plus urgent, plus haletant et sans doute plus tragique le problème d'Hérode» (Scherer 1986: 1319). In tal modo però l'opera di Tristan si discosta dalla sua fonte principale, in cui l'intenzione didattica si concretizza nel racconto di una storia esemplare che illustra le passioni dei protagonisti. La tragedia invece aspira a suscitarle nello spettatore, al fine di ottenerne una maggiore partecipazione affettiva.

Tristan fa iniziare la sua tragedia proprio nel momento in cui nella storia della Cour sainte si inserisce la peripezia: «Enfin arrive un malheureux jour», scrive il P. Caussin (1653: 235). Il primo atto, tutto incentrato sulla narrazione del sogno di Erode, serve a evocare i crimini passati del re, in particolare quelli che colpiscono la casa di Marianna (l'uccisione di Ircano, e soprattutto del fratello Aristobulo); nonché la passione del sovrano per la regina. Le prime scene del secondo atto introducono Marianna. L'azione tragica inizia solamente alla quarta scena dell'atto (con la cacciata di Marianna che continua a disdegnare l'amore del re), e si chiude con l'esecuzione della protagonista dopo la falsa delazione del coppiere e il conseguente giudizio nel quarto atto. Il quinto atto servirà solo a mettere in mostra il delirio di Erode, pentito di aver ordinato l'esecuzione. La concentrazione dell'azione tragica in due atti e mezzo permette di apprezzare il modo in cui Tristan intenta accentuare l'atmosfera tragica; in questo del resto si discosta dal P. Caussin e sembra seguire piuttosto l'altra fonte della storia, La guerra giudaica di Giuseppe Flavio, in cui si narra che Erode «appena sentì che il segreto era stato svelato, uscì fuori di sé [...] impazzito 
dall'irrefrenabile gelosia, ordinò che entrambi fossero immediatamente messi a morte» (1974:179). Questo non è tuttavia l'unico punto in cui l'autore si discosta dalla sua fonte principale.

La visione manichea, caratteristica della Cour sainte, si trova messa in discussione attraverso una maggiore profondità psicologica che manifestano i personaggi di Marianna e soprattutto di Erode. Il carattere monolitico della Marianna di Caussin è stemperato dalle notizie di G. Flavio, che ne fornisce un ritratto meno ideale: assai altiera («Scagliava terribili insulti contro la sorella e la madre di lui [Erode]») ${ }^{7}$, piena di rancore verso Erode piuttosto che paziente («l'odio di Marianna verso di lui era pari all'amore di lui per lei», p. 177), diventando così nella tragedia corresponsabile della propria condanna e dell'infelicità di Erode:

Marianne.- Et moi qu'il a rendu un objet de pitié J'abhorre tout de lui, jusqu'à son amitié (v. 525-526)

Marianne.- Tous les miens sont passés, je brûle de les suivre.

HÉrode.-Comment? veux-tu mourir pour m’empêcher de vivre? (v. 927-928)

Ma ciò che rende la figura di Marianna più umana e meno distaccata è l'amore materno che ritroviamo solo in Tristan, quelle lacrime versate meditando la sorte dei figli che hanno la forza di smorzare l'ira del re:
MARIANNE.- Si je me plains encore d'un arrêt si sévère, C'est à cause que j'ai des sentiments de mère; Je laisse des enfants, et m'afflige pour eux; [...] Elle se porte un mouchoir sur les yeux
HÉrode.- Au point que mon courroux était le plus aigri, Par le cours de ses pleurs mon cœur s'est attendri (v. 867-878)

D'altro canto queste ultime parole segnalano quanto la figura del protagonista tragico sia differente da quella del personaggio del racconto esemplare di Caussin. Nella Cour sainte Erode non condanna Marianna solamente per poter soddisfare ad un amore prettamente egoistico («estimant peut-estre qu'il la rendroit par ce moyen plus souple à sa passion», p. 238), segnalato attraverso la metafora venatoria (p. 222). In Tristan Erode non sembra invece amare «que l'escorce» di Marianna, come asserisce Caussin (p. 221). G. Flavio scrive a proposito che Erode «era perdutamente innamorato», e che «la passione [...] lo struggeva con amore crescente di giorno in giorno» (p. 175-177). In Tristan quest'amore diventa così una divinità mitologica ${ }^{8}$, a cui sacrificare tutto, financo la stessa vita con tutte le sue speciose vittorie, come ammette lo stesso re:

7 G. Flavio 1974: 177. Tristan (1986: v. 275) farà dire a Solomé «qu'elle parle de nous comme de ses valets», e poi alla stessa Marianna «Moi? que je me contraigne? étant d'une naissance / Qui peut impunément prendre toute licence» (v. 355-356 corsivo nostro).

8 J. Morel (1991: 86) ha parlato in questo senso di «"mythologisation" de l'histoire dans les structures». 


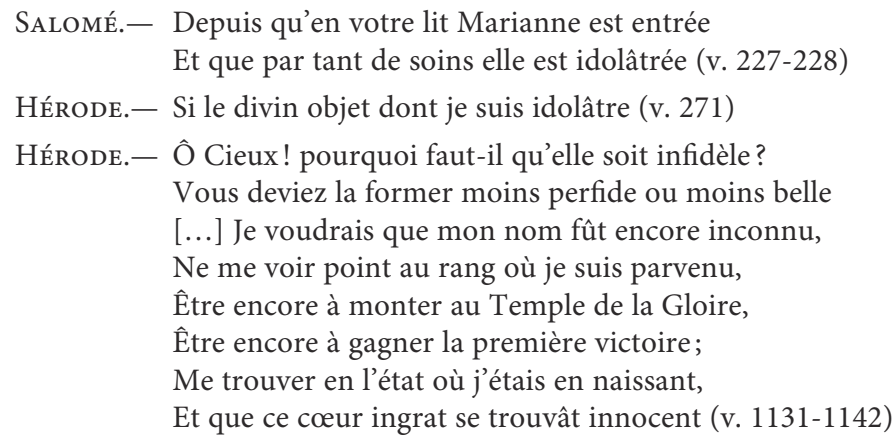

Come ha opportunamente segnalato D. Dala Valle è soprattutto attraverso il personaggio di Erode che Tristan riesce a trasmettere le emozioni tragiche (Tristan 2009: 14): il terrore suscitato da una passione ottenebrante che conduce al delirio; la pietà verso un uomo che agisce incoscientemente per perdersi, e si ritrova infine solo'.

Tutto ciò rende l'atmosfera della tragedia totalmente dissimile a quella che ritroviamo nel racconto del P. Caussin. Le due opere condividono il finale infelice, certo. Tuttavia - come ha illustrato acutamente E. Hénin - la Cour sainte ne suggerisce una spiegazione provvidenzialistica che, proprio attraverso la polisemia della parola "atto» ed il conseguente richiamo alla metafora del theatrum mundi, è volta a giustificare la teodicea cristiana. In tal modo, focalizzandosi sul martirio, l'ultima parola non è lasciata al tragico (Hénin 2007: 115-117). Di fatto per Caussin il delirio di Erode ha cause sovrannaturali, essendo stato «frappé de quelque traict invisible» (p. 239).

In Tristan, all'opposto, la follia di Erode occupa tutto il quinto atto, e tale ipertrofia non è scevra di conseguenze emotive e di accenti tragici (Scherer 1986: 1320-1321). Il drammaturgo riesce così a infondere a una storia originariamente permeata da una visione provvidenzialistica, una visione del mondo più ambigua e problematica. Del resto anche in altre rappresentazioni coeve «si la Providence sert à justifier théoriquement la suite des causes, elle n'est pas à l'origine d'un savoir pratique et ne permet pas de comprendre l'enchaînement logique des événements contingents qui semblent davantage le fruit du hasard» (Zanin 2014: 392). Si aggiunga che l'ingiusto supplizio di personaggi secondari, che pertanto non assurgono allo statuto di martire, come Soême e l'eunuco della regina, aumentano tale sentimento di disordine e di iniqua sorte.

In realtà, a parte la nefasta passione di Erode, è soprattutto la sua follia che sembra segnalare una visione materialistica della morte; presenza che del resto non è estranea né alle scene tragiche, né nella cultura coeva, tanto libertina

9 Il destino di Erode, che rifugge la condanna della regina e reitera costantemente questa sua volontà, sembra per certi versi accomunarsi a quello di Edipo. Entrambi del resto faranno l'esperienza della solitudine davanti al loro destino. 
quanto popolare ${ }^{10}$. Che Tristan sia stato o meno seguace delle idee libertine del maestro Théophile, rimane comunque il fatto che «l'ambiguïté de sa pensée est peut-être le secret de la puissance de son ouvre dramatique» ${ }^{11}$ (Scherer 1986: 1314).

Un dettaglio, infine, può essere utile per apprezzare correttamente la fallace atmosfera che regna nella Mariane di Tristan (ancorchè smorzata dalla cacciata delle due malevoli consigliere nel quinto atto). Allorquando condotta in supplizio la regina assiste agli ipocriti biasimi della madre, Caussin racconta che Marianna replica «par un généreux silence» (p. 238). Tristan trasforma questo silenzio con una arguzia che mette in rilievo quanto il corso delle cose intramondane sia degenerato: «Vous vivrez innocente, et je mourrai coupable» (v. 1392).

Così, lungi dal proporre una storia esemplare, Tristan mette in scena un universo aberrante in cui il Caso o la Provvidenza sembrano gli spettatori di una storia nella quale i personaggi agiscono contro i propri interessi. In questa corsa verso la propria rovina l'annichilimento della libertà umana ha una rilevanza fondamentale. Non a caso è di questo tema che si alimenta essenzialmente il teatro tragico dell'epoca.

\section{La libertà "negativa" nella Mariane: metafisica tridentina, libertà cartesiana e aporie}

In un recente seminario suggerivamo cautamente che, vista l'importanza assunta dal dibattito teologico-filosofico sulla libertà umana nei secoli XVI e XVII, "la comparsa sulle scene europee di numerosi capolavori del teatro tragico potrebbe leggersi in parte come conseguenza dell'enorme quantità di materiale teologico a disposizione successivamente volgarizzato dalla letteratura drammatica coeva, dal momento che, sulla scia della riscoperta della Poetica aristotelica, la tragedia è compresa come "imitazione di un'azione seria [...] e perciò in massima parte di persone in azione [prattontes]"». Pertanto non sarebbe un caso che diversi capolavori drammatici coevi, attraverso il loro intrigo, abbiamo posto incessantemente tale problema in primo piano (Reina Li Crapi à paraître). Del resto nel volume monografico che Littératures classiques ha dedicato alla "périodisation de l'âge classique», D. Souiller e B.

10 Quanto al teatro e alla cultura libertina citiamo a titolo d'esempio la celebre pointe di Cyrano nella Mort d'Agrippine: «Térentius: Qui les craint [les dieux], ne craint rien. Séjanus: Ces enfants de l'effroi, / Ces beaux riens qu'on adore, et sans savoir pourquoi / [...]. Va, va, Térentius, qui les craint, ne craint rien» (Cyrano 2005: 44). La presenza di visioni materialiste nella cultura popolare dell'Europa moderna è stata ben evidenziata da C. Ginzburg attraverso l'esempio di Menocchio visione che ritroviamo a fine Xvil secolo all'interno del clero, come prova Le Testament di J. Meslier.

11 In Tristan (2009: 11), scrive D. Dalla Valle, «se mêlent deux exigences différentes, je dirais même opposées, qui cherchent un équilibre sans l'atteindre toujours: d'une part, il y a le souvenir de l'expérience libertine, vécue par Tristan dans sa première jeunesse, mais désormais éloignée et étouffée; de l'autre, il y a la présence d'une expérience religieuse, qui voudrait s'imposer sur la première». 
Chédozeau hanno segnalato la fondamentale importanza del fattore religioso e la presenza di una spiritualità gesuitica nella letteratura francese nella prima metà del secolo.

D'altro canto il libero arbitrio postulato dalla dottrina molinistica non potrebbe che essere messo in discussione in una rappresentazione tragica, in cui l'uomo si ritrova spesso schiavo di un destino che sembra ineluttabile. Erode constata con amarezza all'inizio della tragedia di Tristan che

Ce qu'écrit le destin ne peut être effacé.

Il faut bon gré mal gré, que l'âme résolue

Suive ce qu'a marqué sa puissance absolue.

De ses pièges secrets on ne peut s'affranchir,

Nous y courons plus droit en pensant les gauchir (v. 146-150)

Come esprimere dunque la libertà dell'uomo in un genere teatrale in cui il protagonista si riconosce impotente di fronte agli avvenimenti a cui deve far fronte, e sottomesso a un destino ch'egli crede già prestabilito? In realtà nella tragedia di Tristan il personaggio di Marianna - una coscienza religiosa - afferma che «[sa] mort est à la fois contrainte et volontaire» (v. 1316), dal momento che è la sua "envie [qui] travaille puissamment à creuser [son] tombeau» (v. 1247).

In generale quanto è stato scritto a proposito della relazione tra Cartesio e Corneille può essere in larga parte ricondotto ad altri drammaturghi coevi ${ }^{12}$. In Cartesio l'atto fondamentale del cogito nello stesso tempo in cui fornisce una prova inconcussa dell'esistenza umana, assicura anche la certezza di una libertà interiore. Essa è dunque innanzitutto una libertà di pensiero, un carattere inerente della res cogitans; ragion per cui non potrebbe essere alienata a causa di fattori esterni, come testimonia dalla prima fino all'ultima comparsa il personaggio di Marianna:

Si mon corps est captif, mon âme ne l'est pas (v. 362)

Mais en quelque péril que le malheur m'engage,

J'aurai cet avantage

Que mon cœur pour le moins se rendra le dernier (v. 1242-1244)

Che la libertà possa solo essere alienata a causa di disposizioni interne all'animo umano può del resto essere dedotto dalla riscrittura della prima scena: mentre Hardy chiamava in causa nella sua omonima tragedia un fantasma, ossia un fattore esterno, Tristan opta per un sogno spaventoso che scuote l'animo del re («mon esprit est troublé du songe que j'ai fait» v. 26); seguendo verosimilmente l'esempio fornito poco prima dalla rappresentazione della Mort de César di Scudery (Morel 1991: 206).

12 «Les poètes de cette époque ont en commun non seulement un vocabulaire, mais aussi une culture mythologique, historique et biblique, et des préoccupations éthiques et métaphysiques qui les font s'interroger sur la nature et la condition de l'homme» (Morel 1991: 33). Questa idea era già stata suggerita da E. Cassirer: «[Lanson] cherche à l'expliquer par leur commun “entourage". C'est bien plutôt leur attitude vis-à-vis de l'ensemble de l'existence qui manifeste une affinité intime» (1997: 19). 
Da un lato, quindi, le parole di Marianna, il cui esempio appare come una trasposizione drammatica della terza massima che Cartesio enuncia nel Discours de la méthode, secondo la quale l'uomo può disporre liberamente soltanto della sua volontà, poiché «il n'y a rien qui soit entièrement en [son] pouvoir que [ses] pensées» (Descartes 2000: 100). In questo modo, avendo acquisito "ce pouvoir absolu sur nos pensées», scrive N. Grimaldi parafrasando Cartesio, persino «l'homme le plus puissant du monde y sera moins puissant que nous» (Grimaldi 1988: 59). Il rinvio al controesempio offerto da Erode che si lamenta della propria condizione è inevitabile. Esso mette in evidenza, dall'altro lato, una padronanza di sé, e conseguentemente un grado di libertà, diametralmente opposto a quello espresso dalla regina (v. 205-224).

Eppure anche in Erode è possibile reperire una parvenza di libertà attraverso le sue parole, le quali manifestano sprazzi di lucidità,

L'homme à qui la Fortune a fait des avantages,

Est comme le vaisseau sauvé de cent orages,

Qui, sujet toutefois aux caprices du sort,

Peut se perdre à la rade, ou périr dans le port (v. 151-154)

L'amour est tellement fatal à la valeur

Qu'il n'est point de héros exempts de ce malheur (v. 245-246)

J'interromps cet arrêt, car ma colère extrême

Te faisant ton procès, me le fait à moi-même,

Et si dans un moment je n'arrêtais ton deuil,

Je sens bien qu'avec toi j'irais dans le cercueil (v. 899-902)

Tuttavia questa lucidità è «tragiquement inutile»(Scherer 1986: 1321). Così ad esempio malgrado il protagonista sia cosciente che

Nous ne pouvons jamais, avecque bienséance,

Aux rapports des valets, donner tant de créance

[...] Et même le mensonge est assez ordinaire

À ces petites gens dont l'âme est mercenaire (v. 303-308)

poco dopo sarà ingannato dalla falsa delazione del suo coppiere. Per Caussin la protagonista viene condannata al supplizio durante il processo che segue. Invece Tristan modifica il dato, ponendo l'accento sul fatto che la decisione fatale è presa non durante un giudizio, bensì in un momento di completo oscuramento della ragione a causa della gelosia, così come giustificato da G. Flavio:

Appena sentì che il segreto era stato svelato, uscì fuori di sé, e considerando che Giuseppe [Saône nella tragedia n.d.r.] non le avrebbe rivelato l'ordine ricevuto se non per averla sedotta, riarse di sdegno e, balzato giù dal letto, prese a vagare qua e là per la reggia. [...] Erode, impazzito dall'irrefrenabile gelosia, ordinò che entrambi fossero immediatamente messi a morte (p. 179).

Tristan vuole mettere maggiormente in risalto il carattere estemporaneo della condanna: se è nella durata che una libera scelta può realizzarsi ${ }^{13}$, la

13 «L'acte libre se produit dans le temps qui s'écoule, et non pas dans le temps écoulé» (Bergson 1991: 166). 
passione amorosa, insoddisfatta, sembra svuotare di valore il tempo umano e rendere l'universo del protagonista illusorio. La tragedia suggerisce che per essere veramente libero Erode dovrebbe rinunciare alla sua passione. Orbene, questa libertà che, come in Cartesio, può essere raggiunta solamente attraverso la negazione dei desideri - dato che una delle potenzialità della volontà umana è quella di rifiutare tutto - appare in qualche modo paradossale come ha notato J.-P. Sartre ${ }^{14}$. Essa sembra tuttavia far eco alla formulazione negativa con cui il quarto canone della sesta sessione del concilio tridentino suggella il 13 gennaio 1547 il dogma del libero arbitrio:

Se qualcuno dice che il libero arbitrio dell'uomo, mosso ed eccitato da Dio, non coopera in nessun modo esprimendo il proprio assenso a Dio, che lo muove e lo prepara ad ottenere la grazia della giustificazione; e che egli non può dissentire, se lo vuole, ma come cosa senz'anima non opera in nessun modo e si comporta del tutto passivamente: sia anatema (citato in Schmutz 2002: 58, corsivo nostro).

Ma se il libero arbitrio umano si riduce «alla capacità di dire no» (Schmutz 2002: 57), se la libertà si può raggiungere solo attraverso la rinuncia a tutto quello che è immanente, allora nessun personaggio potrebbe definirsi totalmente libero nelle scene francesi. Persino quando si sottostima la propria vita, e ci si appresta deliberatamente ad affrontare la morte come nel caso di Marianna, in verità giammai si rinuncia completamente alle aspirazioni intramondane. La regina si mostra difatti tanto attaccata all'amore per i figli (v. 1321-1336) quanto al passato deplorevole della propria famiglia (v. 379-390) ${ }^{15}$.

Il contesto intramondano, l'immanenza, rappresenta dunque un limite per una volontà «infinita quodammodo dici potest» ${ }^{16}$. Limite che si concretizza nelle situazioni particolari in cui si trovano ad agire i personaggi; in particolare allorquando uno di essi si trova in uno stato che Cartesio definirebbe di «indifférence» negativa. In tali situazioni, infatti, «la volonté se détermine avec précipitation selon des perceptions obscures et confuses de l'entendement», oppure «relâche l'attention qui l'attachait aux perceptions claires et distinctes de l'entendement» (Bouchilloux 1997: 79-80). In altre parole l'incostanza della volontà causa una forma di negligenza che comporta uno svilimento della libertà

14 N. Grimaldi, riprendendo i due paradossi evidenziati da Sartre (p. 69-70), scrive a proposito: «soit qu'on considère l'une, soit qu'on considère l'autre, nous avons vu la liberté n'y être tantôt qu'abnégation, tantôt que négation. "Étrange liberté", peut donc bien dire Sartre. Tantôt on la croit positive, mais elle se réduit à une simple adhésion qui ôte à la volonté jusqu'à son autonomie, si bien que de la liberté ne reste plus que le nom. Tantôt, la volonté récupère bien son autonomie, mais réduite à nier le néant, la liberté y est si peu que rien» (Grimaldi 1988: 76).

15 Un discorso simile può essere fatto per Sofonisba e Massinissa nella tragedia di Mairet, o di Deianira, Iole e Arcas nell'Hercule mourant [1634] di Rotrou: tutti sono pronti a sacrificare la propria vita, ma non il loro amore. Nell'Horace [1640] Camille non saprà rinunciare a Curiace (III, iv, v. 917-927) e Sabine rimarrà in una straziante impasse non volendo rinunciare a niente (III, i, v. 711-764).

16 R. Descartes, Principes, citato Grimaldi 1988: 25. Sulla questione si veda tra gli altri J.-B. Jeangène Vilmer 2008. 
umana nella vita pratica, conducendo l'uomo all' «erreur théorique» o alla «faute morale» (Bouchilloux 1997: 80). L'esempio offerto da Erode nella Mariane sembra coincidere ancora una volta con le speculazioni cartesiane. Le parole con cui Salomé incoraggia il coppiere a fare la falsa delazione testimoniano bene dello stato di «indifférence» negativa del protagonista:

Ton rapport va surprendre une âme défiante,

Crédule, furieuse, et fort impatiente.

Dans ce trouble excité, si tu fais ton devoir,

Il mordra l'hameçon sans s'en apercevoir (v. 583-586)

Durante il processo, benché Erode si proponga di stabilire la verità invitando il coppiere a reiterare le accuse di fronte alla regina, si ritroverà ingannato, credendo Marianna colpevole di attentare alla sua vita. Tuttavia, commosso dalle lacrime della regina, sarà disposto immediatamente dopo a perdonarla. Come già accennato, è significativo che in questo caso Tristan preferisca non seguire Caussin, scegliendo di non far condannare Marianna durante il giudizio (v. 899-902). Tale atteggiamento segnala infatti la presenza en amont di una certa forma di libertà. Il brusco cambiamento della personalità che secondo Cassirer caratterizza molte delle creazioni cornelliane, lo ritroviamo così anche nell'Erode di Tristan, a indicare la presenza di una volontà che rimane malgrado tutto inestinguibile:

De telles transformations subites précisément ne sont pas possibles que parce que les hommes de Corneille ne sont pas livrés, sans plus, à leurs passions, mais qu'ils les affrontent avec une force opposée, la force de la réflexion sur soit et du jugement. Quand le jugement leur propose un nouveau but et que leur connaissance se modifie, la volonté suit aussi ces modifications ${ }^{17}$. (Cassirer 1997: 12-13)

Tuttavia la passione amorosa è capace di offuscare totalmente la ragione e annichilire la volontà. Il carattere pernicioso delle passioni era già stato utilizzato da Agostino d'Ippona per giustificare metafisicamente l'esistenza delle cattive azioni ${ }^{18}$. Sarà appunto la gelosia a creare la situazione di cui Salomé potrà

17 Dopotutto anche la concezione molinistica della libertà con il suo sensus compositus insiste sul fatto che «un agente agisce liberamente quando date tutte le condizioni richieste per l'azione, può sia agire che non agire, o che agendo in un certo modo, può anche agire in senso contrario», L. de Molina, Concordia, citato da Schmutz 2002: 60.

18 «E chiaro che soltanto la passione è determinante del generale concetto di azione malvagia», (Agostino: I, iii, 8). Tornando sull'argomento all'inizio del III libro, Evodo domanda: «vorrei conoscere da te da chi proviene quel movimento per cui la volontà si muove in senso opposto al bene universale e non diveniente e si muove verso i beni particolari, estranei o infimi, tutti divenienti [...] di chi è questo movimento che ritieni certamente colpevole?». Agostino risponde: «è stato [già] accertato che soltanto con la propria volontà la coscienza diviene schiava della passione e conseguentemente che non può subire costrizione a tale stato di abiezione né da un essere superiore oppure eguale perché sarebbe ingiustizia, né da un inferiore perché esso non ne sarebbe capace. Rimane dunque che sia suo personale questo movimento, con cui volge dal Creatore alla creatura la volontà di godere. Quindi tale movimento, se si deve attribuire a colpa, non è naturale ma volontario» (III, 1-2). 
approfittare - Tristan ritorna così a seguire La Cour sainte - strappando dalla bocca del re quel "qu'on l'ôte» (v. 1232) estemporaneo che condanna Marianna. Non è un caso del resto che la maggior parte delle colpe tragiche nelle scene francesi abbia il carattere di un gesto irriflesso: l'azione tragica è messa in moto (Sophonisbe, Cid) o conclusa (Mariane, Horace) da un gesto avventato. Corneille utilizza proprio l'espediente del "premier mouvement», prima di sperimentare l'azione "entravée» in Cinna (Forestier 2004: 116), per rendere conto della cattiva azione di un personaggio. La condanna del gesto irriflesso non comporta tuttavia una disapprovazione generale dell'azione. Come per la «morale provisoire» cartesiana, anche sulla scena la prontezza dell'azione si rivela all'occorrenza indispensabile.

Nelle tragedie francesi ritroviamo dunque due tipi di libertà, di parola/ pensiero e di azione, che evocano, in particolare in Corneille, due prototipi differenti di magnanimità. Il primo tipo è riconducibile alle parole e riflessioni di quelle che S. Dubrovsky ha definito «existences féminines» (1982: 186). Esso manifesta l'eroismo di certi personaggi nella misura in cui, benché passivamente (ma comunque sostenuti dal proprio orgoglio) li mette «à l'abri de l'humiliation», permettendo loro di ottenere «des victoires idéales plus précieuses que le succès matériel» (Bénichou 2011: 29). Nella prospettiva religiosa che l'accomuna alla sua fonte, il martirio di Marianna è senza dubbio una vittoria sulle avversità del mondo e sulle sue tirannie: una vittoria della libertà, come lo sarà più tardi anche quella dell'Edipo di Corneille.

La libertà d'azione, su cui torneremo fra poco, è più difficile da discernere. Se in effetti l'esito di un'azione non indica necessariamente che essa sia stata compiuta spontaneamente, dall'altro lato i personaggi sembrano agire in maniera libera soprattutto quando aderiscono a un ordine superiore (lo Stato, Dio). Cosicché gli «esprits mâles», la cui libertà si esprime attraverso le azioni intraprese, si credono liberi solamente se - o fino al momento in cui - non portano una riflessione approfondita sulla loro condizione, o non sperimentano l'insuccesso ${ }^{19}$.

Riassumendo, per Cartesio e per i drammaturghi coevi la riflessione e l'azione rappresentano due momenti fondamentali in cui è espletato l'esercizio della libertà. Con la differenza che questi ultimi ereditano tutta una serie di situazioni tragiche che mirano a diminuire la libertà d'azione. Da questo punto di vista l'unità di tempo concorre a rendere i contesti in cui il personaggio opera più insidiosi, riducendo le possibilità di incidere efficacemente sulla propria sorte, e non lasciando loro che il fiato per biasimare gli dei o il destino. Se una vera libertà scaturisce solamente dalla concomitanza di una coscienza dilaniata dall'impasse tragica, e dalla riuscita dell'impresa che la sua volontà

19 Un esempio emblematico di questa libertà è offerto dal personaggio di Augusto in Cinna, che afferma alla fine della tragedia: «Je suis maître de moi comme de l'Univers: / Je le suis, je veux l'être. Ô siècles, ô mémoire, / Conservez à jamais ma dernière victoire» (Corneille 1980: v. 1696-1698). 
s'era prefissata di compiere, allora è possibile affermare che le scene tragiche mostrano raramente degli spiriti veramente liberi.

Invero a minacciare il personaggio sulla scena è soprattutto il tempo. Nella Mariane Erode è permanentemente cosciente che un'eventuale condanna di Marianna gli negherebbe per sempre ciò che la sua volontà desidera con più ardore, e conseguentemente dell'inanità del suo potere («ma gloire n'est qu'un songe, et ma grandeur qu'une ombre» v. 224). Ragion per cui, creduta l'accusa del veleno che Marianna tramava di fargli bere, desidera, come abbiamo visto, piuttosto tornare indietro nel tempo che accettare tale realtà: (v. 1137-1142). Successivamente, durante l'ultimo atto, si urterà ancora più tragicamente con l'irreversibilità del tempo, ordinando insistentemente di far comparire Marianna che ha appena condannato a morte (v. 1671-1673, 1736-1740)

In questo modo è l'impotenza del re che è messa in primo piano. La volontà umana si scopre pertanto nel suo più alto grado di debolezza; la sua «infinitude» non è allora altro che il segno di una mancanza ${ }^{20}$, di cui solo una parola, sprovvista oramai di senso, attesta la presenza. La libertà di Erode, dipendendo totalmente da un personaggio di cui «son nom seul est resté» (v. 1751), si rivela essere in fin dei conti solo un'idea lontana da ogni realizzazione concreta: un'astrazione del pensiero, un'idea universale che è soltanto realtà mentale, senza alcuna materializzazione all'infuori di ciò. Insomma un nome, quel solo nome che gli è rimasto, flatus voci.

\section{Horace e l'etica di regime}

\section{Dagli exploit di Rodrigo al sacrificio di Alcionée}

Non si possono comprendere esattamente i sentieri intrapresi dalla tragedia francese all'epoca di Richelieu senza tener conto, oltre che delle speculazioni teoriche che inflettono la direzione della vita teatrale coeva, dello strepitoso successo ottenuto da rappresentazioni che sono vere e proprie pietre miliari, e influenzano conseguentemente la scrittura delle pièce successive: La Sophonisbe (più che l'Hercule mourant di Rotrou), La Mariane (punto d'approdo di tentativi tragici sperimentati con successo la stagione precedente - Médée, La Mort de César, La Mort de Mitridate), Le Cid (ancorché all'incrocio di generi, ma la cui influenza sulla produzione tragica posteriore è determinante, come testimonia ampiamente l'Alcionée di Du Ryer) e poi Horace. Per comprendere l'evoluzione della produzione drammatica sarebbe allora necessario tenere in mente quel contesto, tentare di evocare se possibile il ninot de neu (Massip 2009),

20 N. Grimaldi scrive a proposito di Cartesio: «Lorsque nous disons que le projet cartésien est le négatif de l'ontologie cartésienne, c'est au sens où le désir est le négatif du désiré, au sens où tout désir, étant l'absence de quelque présence, postule comme négatif la présence de cette absence» (1988: 96). 
immaginandosi spettatori - nel parterre del Marais ${ }^{21}$ - i cui sguardi pendono dai gesti e dagli accenti di Mondory ${ }^{22}$.

Le Cid segue La Mariane e per certi versi sembra averne adombrato la fama. Ma perché condannarlo allora? Si è spesso cercato di far chiarezza sulla questione speculando in maniera pertinente e approfondita sulle ragioni estetiche della condanna. Tuttavia le esitazioni di Chapelain, le sue lettere a Balzac dell'estate 1637, l'ordine espresso di Richelieu di pubblicare una condanna esemplare, dovrebbero spingere a dar un maggior peso alle motivazioni etico-politiche della condanna.

Costatiamo innanzitutto che l'esempio del Cid permette di cogliere quella libertà d'azione sovente limitata nelle scene tragiche. Invero, l'opera presenta da un lato un nouement tragico, in cui gli avvenimenti precipitano senza che nessun personaggio possa intervenire per evitare il peggio, con un subitaneo capovolgimento della situazione (Chimène dirà "Que nostre heur fust si proche \& si tost se perdist!» Corneille 2001: v. 998). Dall'altro, il dénouement si caratterizza per un tempo che inizia a correre meno velocemente, contrastando con l'incedere torrenziale dei primi atti. Ciò permette al protagonista di portare a termine con successo le sue imprese; o, per parafrasare una delle accuse mosse alla pièce, si comincia ad avere così tanto tempo che l'autore può ammassarci un numero considerevoli di avvenimenti.

In questo senso, malgrado la condanna del gesto irriflesso che ritroviamo nell'ammissione di colpa dello stesso conte ("J'eus le sang un peu chaud, \& le bras un peu prompt» v. 353-354), l'esempio del Cid mostra quanto sia utile la prontezza nelle situazioni a cui Rodrigo deve far fronte. Del resto lo stesso Cartesio nel Discours de la méthode disapprova l'inerzia e formula una «morale provisoire» che possa essere seguita malgrado non assicuri il buon esito finale. La sua seconda massima prescrive "d'être le plus ferme et le plus résolu en mes actions que je pourrais» per poter agire prontamente quando le circostanze esterne lo richiedono; e di superare le esitazioni anche nelle situazioni più difficili da giudicare (Descartes 2000: 99-100). Se questa massima ha lo scopo di descrivere l'azione libera, i successi di Rodrigo ne rappresentano una riuscita trasposizione drammatica. Alla fine del primo atto il protagonista si trova di fronte a una impasse tragica («misérable vengeur d'une juste querelle», v. 295), proprio come un altro celeberrimo personaggio tragico, Amleto. Gli exploit

21 Il riferimento al gusto del pubblico - checché ne dicano i dotti - è e sarà anche dopo la Querelle sempre evocato. Si consideri inoltre che un'opera come l'Horace, potrebbe essere letta come una provocatio ad populum di Corneille, "une réinstruction du procès du Cid. [...] Interdit de répondre à leurs "sentiments", Corneille fait une seconde tragédie [...] et la soumet au public et, comme pour Le Cid, le peuple, le succès public, lui donnent raison» (Merlin 2009: 112).

22 «Nous devons cela à Jason [Médée], à Massinisse [La Sophonisbe] et à Brutus [La Mort de César] qui vient aujourd'hui en la personne de l'homme dont vous me parlez si avantageusement et que j'ai admiré autant de fois que je l'ai ouï» Lettre di Balzac a Boisrobert del 3 aprile 1635, citata da G. Couton (Corneille 1980: 1376). 
del protagonista iniziano con il superamento di tale situazione (v. 341-342) e con la determinata esecuzione di quanto la sua volontà si è proposto di fare, benché né la soluzione appaia totalmente giusta (v. 1073-1074), né l'esito possa dirsi scontato (v. 415-416). È attraverso l'azione infatti che Rodrigo conferma il suo valore e conseguentemente il suo ruolo nella società: una condotta che trova la sua formula emblematica nel «marchons sans discourir» (v. 441) che il protagonista ingiunge al Conte. Per molte creazioni cornelliane, così come per la morale cartesiana, quello che conta è soprattutto agire dopo essersi determinati a seguire ciò che si crede giusto; come farà del resto anche Polyeucte qualche anno dopo. Da questo punto di vista il successo terreno di un'impresa ha un valore relativo.

Le Cid ci mostra un universo in cui la retorica non è sufficiente a conservare il proprio ruolo nella società; essa è infatti obliterata continuamente dalla «suprématie impitoyable du présent» che comporta «l'annihilation de l'être par la durée» (Dubrovsky 1982: 90). In altre parole l'uomo nel Cid è rappresentato in balia di un tempo che smentisce costantemente le sue parole. Tuttavia con una sola, unica, eccezione: Rodrigo. Egli, che peraltro è pronto a sacrificare la sua vita - e dunque il suo appoggio allo Stato - all'amore di Chimène, si rende sempre scusabile grazie soltanto alle sue imprese. Non permette pertanto di cogliere all'interno della pièce la traccia di un potere riconosciuto come assoluto. Un potere che lo perdoni in maniera del tutto gratuita; o all'inverso che lo condanni perché tale è la sua volontà (mala quia prohibita). La sua figura occulta tale presenza che dovrebbe obbligare incondizionatamente a una sottomissione cieca e deferente. Bisognava quindi condannare il Cid! (punire in un certo senso il suo orgoglio e la sua simpatica guasconeria). Ciò che arrivò non appena Rodrigo uscì di scena.

L'esempio della perentoria condanna del Cid sembra essere stata ben assimilata dai drammaturghi coevi. In realtà già nell'Alcionée di Du Ryer ritroviamo l'ineluttabile influenza del successo di Corneille (Scherer 1986: 1280) coniugata con le esigenze di una volontà politica che non deve essere apertamente mostrata, né però dimenticata: «pièce politique exaltant l'intangible toute-puissance des rois et condamnant le révolté, même prestigieux, elle devait plaire aux gouvernements» (Scherer 1986: 1281). L'esempio di Du Ryer è del resto veramente pregnante se si considera che il drammaturgo ha fino ad allora composto più che altro tragicommedie che veicolano i valori «feudali» dei grandi signori del tempo come César de Vendôme. Questa tragedia che «a plus à Son Éminence», come rivela l'autore nella dedicace (Du Ryer 1986: 85), è invece ora offerta alla duchessa d'Aiguillon, nipote dello stesso Richelieu.

L'influenza del Cid - e delle rappresentazioni tragi-comiche in generale - la ritroviamo innanzitutto nella materia scelta e nel modo di trattarla. L'argomento è preso dal canto XXXIV dell'Orlando furioso, ma Du Ryer cambia un dato fondamentale: l'eroina ama Alcionée, diversamente dalla «bella, ma altiera più» Lidia di Ariosto (1990: 866). Questa inversione da un lato «réalise le passage du romanesque au pathétique» (Scherer 1986: 1282), testimoniando ancora una 
volta la necessaria presenza del tema amoroso nelle scene tragiche francesi, sulla scia del successo di Théophile du Viau:

Lydie.- $\quad$ que fais-je, malheureuse? oublierai-je qu'il aime?

Détruirai-je un Amant? me perdrai-je moi-même? (v. 173-174).

Alcionée.- Allons, fuyons, et sortons de ces lieux

Où trop cruellement me poursuivent les Cieux [...]

Je fuirai; mais enfin ce sera de la vie,

Et je saurai passer avec un noble effort

Des prisons de l'Amour aux prisons de la Mort (v. 1333-1342).

Dall'altro permette di riportare sulla scena un amore contrastato dal dovere, al pari di quello di Rodrigo e Chimene, come ricorda il re (v. 407-408), ma soprattutto come spiega fin dai primi versi l'eroina:

Lydie.- Moi, je pourrais souffrir l'amour d'Alcionée!

Un amour qui m'outrage, et qui m'a ruinée (v. 1-2).

L'identificazione tra Alcionée e Rodrigo è del resto esplicitata pochi versi dopo dalla confidente della principessa che riferisce che l'eroe

peut être d'un trône ou la base ou l'effroi,

Et qui pour vaincre tout n'a besoin que de soi (v. 15-16, corsivo nostro)

così come più tardi dalle parole del re che riconosce nel protagonista «une âme non commune» (v. 598), e dalle parole stesse del protagonista che rinviano letteralmente a una celebre scena del Cid:

Si vous me condamnez, si mon trépas vous plaît,

Donnez, donnez le coup aussitôt que l'Arrêt (v. 965-966)

Ma nella tragedia di Du Ryer, e a fortiori dopo la Querelle, un nuovo Cid non può più affermarsi sulla scena con il solo aiuto delle proprie forze. Rodrigo si era guadagnato il perdono senza l'ausilio di nessuno; nel Cid non ritroviamo la magnanima clemenza di un re che fa un violento strappo allo stato di diritto. Alcionée, il cui norme ricorda fra l'altro la scalata dei giganti all'Olimpo (v. 126 e 914), è ora nella tragedia di Du Ryer destinato a una fine tragica:

Roi.- Soyez en vos desseins plus juste et plus modeste Quand l'espoir est trop haut, il est souvent funeste (v. 537-538) 23

Tuttavia, per ammissione dello stesso re, «quand il aimerait, en serait-il coupable ?» (v. 457). L'amore rimane un tema centrale dell'opera, e la situazione tragica è data anche dal fatto che esso non sarebbe di per sé condannabile - e non potrebbe esserlo altrimenti, tenuto conto della dedica ${ }^{24}$. La tragedia di Du Ryer

23 L'ingratitudine diventa così il simbolo del potere reale. Del resto ognuno di noi, verosimilmente, ha provato qualche volta l'esperienza di vedersi rifiutare una parte di qualcosa che aveva precedentemente domandato senza che ci fosse un motivo veramente ragionevole; o meglio, con l'amara sensazione che tale parziale diniego sia dovuto alla mera volontà di esplicitare il potere. In questo senso Du Ryer avrebbe potuto trarre spunto per il suo soggetto dai versi dell'ottava 27 del canto, in cui viene presentato un re che «né mai si piega alla prima richiesta» (Ariosto 1990: 889).

24 Rinviamo alla lettura di Mirame fatta da G. Couton (1986: 57). 
si configura quindi come "poème de l'ingratitude» (Scherer 1986: 1283): se la pièce non può che piacere a Sua Eminenza, dal momento che sembra suggerire la legittimità di un amore ambizioso, dall'altro manca in essa una volontà onnipotente che testimoni della sua bontà. Dopo il Cid, la tragedia di Du Ryer forse non avrebbe potuto metterla in scena.

\section{La necessità della colpevolezza}

Non è un caso che le due tragedie successive di Corneille abbiano per soggetto una storia che permetta di «justifier cet acte inouï qu'est le pardon du crime d'Horace» (Forestier 2004: 85), e più tardi di Cinna. Come testimoniano la lettera di Chapelain a Balzac del 15 gennaio 1639 e il silenzio delle scene, la condanna del Cid segna profondamente la produzione drammatica dell'autore. Egli non torna a scrivere per il teatro prima di aver passato due anni a riflettere su questioni di poetica. Pur non sottovalutando l'importanza della politica, della brodérie, nelle opere di Corneille (Forestier 1998: 68), G. Forestier ha mostrato che le motivazioni poetiche sono primordiali nelle scelte dell'autore; pertanto l'Horace non rappresenterebbe «l'entrée en politique» (Forestier 2004: 111). Modificando l'accezione che viene data alla "politique» possiamo dirci d'accordo dal momento che pensiamo che l'entrée sia già avvenuta per lo meno nel 1637, all'indomani dello scoppio della Querelle. Che ne sia stato cosciente o meno, al tempo di Richelieu, riprendere la piuma è già di per sé un atto politico ${ }^{25}$ - e lo sarà anche successivamente, come ha dimostrato J.-P. Sartre in Qu'est-ce que la littérature?. In questa prospettiva il contenuto politico dell'opera è implicito nella fase di inventio, nella scelta del soggetto (e non di un tema).

Corneille quindi ritorna a scrivere al fine di «plaire pour divertir, divertir et ainsi servir l'État» (Jouhaud 1994: 137), scegliendo una storia in cui un eroe, al culmine della gloria, commette un crimine terribile, a cui solo una voluntas absoluta potrebbe concedere il perdono. Così, «face aux doctes qui ont critiqué l'inventio - le meurtre délibéré et sous les yeux du public de Camille par Horace -, Corneille émet des réserves sur la dispositio [...] Comment dire autrement que c'est précisément pour cela qu'il avait choisi ce sujet?» (Forestier 2004: 75-77).

G. Couton ha segnalato le fonti della tragedia, indicando il senso della riscrittura della storia di Tito Livio fatta da Corneille appoggiandosi sull'opera di Dionigi d'Alicarnasso (Corneille 1980: 1540-1543). Più recentemente la brillante lettura di $\mathrm{H}$. Merlin ha messo in rilievo come, riscrivendo l'atto fratricida di Orazio, Corneille abbia trasferito la scena del crimine dal luogo pubblico alla casa privata, modificandone dunque il valore (2009: 109). Ancora più importante è la modifica delle conseguenze giudiziarie: la tragedia infatti sostituisce alla provocatio ad populum - istituzione repubblicana - il giudizio del re. Il popolo assolve Orazio secondo la legenda; ma nella Francia di Richelieu tale assoluzione non ha più un valore determinante. Il ruolo del pubblico

25 Politico non solo perché atto pubblico, ma perché s'inserisce nella - o collide con - la politica culturale di Richelieu. 
dunque - ed è questa per H. Merlin un'ulteriore giustificazione della scelta del soggetto - prende valore contro i duumviri solo nella misura in cui è prima, «à l'intérieur du cabinet», che il cardinale ha dato il suo assenso.

In altre parole è lo stesso potere politico che detta e giustifica le regole dell'arte e se ne compiace, come ha scritto C. Jouhaud: «les secrets de l'art surgissent des secrets du pouvoir saisis à travers les effets du secret de l'art» (1994: 141). A questo punto appare inevitabile il rinvio ai primi libri della Repubblica, in cui Socrate spiega l'importanza di utilizzare il discorso che fa ricorso alla finzione per educare i giovani (Platone 2009: 305, 375), dal momento che il filosofo suggerisce che «la prima cosa da fare è tenere d'occhio gli ideatori delle favole: quando ne inventassero una bella la approveremo in caso contrario la scarteremo» (Platone 2009: 307).

Ritorniamo alla poetica di Corneille e alla sua autocritica dell'Horace:

Cette action, qui devient la principale de la Pièce, est momentanée et n'a point cette juste grandeur que lui demande Aristote [...] le second défaut est que cette mort fait une action double par le second péril où tombe Horace (Corneille 1980: 840)

Per il drammaturgo il difetto più grave della pièce sta proprio nell'inventio, dal momento che presenta una doppia azione - aggravata peraltro dalla loro dispositio. Come potrebbe un drammaturgo attento alla teoria come Corneille, che peraltro «a achevé son éducation théorique à ce moment-la» (Forestier 2014: 112), in una tragedia che si vuole fermamente rispettosa delle regole, commettere un errore così grossolano? Abbiamo visto che ciò che giustifica la scelta del soggetto, di questa «œuvre hybride», non è l'azione tragi-comica ma la seconda azione; lo scopo essendo quello di perdonare un gesto nefando, e mettere «ce généreux Romain [...] aux pieds de Votre Éminence» (Corneille 1980: 833).

Quanto alla prima, invece, sarebbe possibile che il drammaturgo abbia deciso di non prescinderne dal momento che si tratta della trasposizione scenica dell'esemplare comportamento del difensore della città platonica?

«Un solo pugile che sia perfettamente preparato all'incontro, non ti sembra che avrebbe vita facile a liquidare due avversari ricchi e panciuti?» «Forse no, se i due scendono in campo assieme» obiettò. "Neanche - domandai - se gli permettessimo di simulare una fuga e all'improvviso di voltarsi e colpire il primo che di volta in volta gli si para innanzi? E se questa mossa la ripetesse più e più volte sotto il sole e la calura estiva non diresti che un pugile siffatto potrebbe stendere più di uno di tali avversari?» (Platone 2009: 449).

\section{Conclusioni}

Nella Repubblica Socrate decide di bandire i poeti poiché l'imitazione può avere effetti perniciosi. Secondo R. Girard (2017: 217) infatti «le désir est essentiellement mimétique, il se calque sur un désir modèle [...] Toute mimesis portant sur le désir débouche automatiquement sur le conflit». D’altro conto 
il crimine d'Orazio, dopo quello evocato di Romolo (v. 1756), potrebbe anche leggersi come un «sacrificio» ineluttabile dal momento che «le sacrifice empêche les germes de violence de se développer», e che "plus la crise est aiguë, plus la victime doit être "précieuse"» (Girard 2017: 33)?

Come rende manifesto quest'ultimo abbozzo di riflessione le nostre analisi si sono avvalse di interpretazioni per un certi versi divergenti. Tale ricorso è giustificato dal fatto che, da un lato, «toute œuvre qui compte, dans la littérature dramatique du $\mathrm{XVII}^{\mathrm{e}}$ siècle, est réécriture à la fois d'une autre grande œuvre antique (théâtrale ou non) et d'une ou plusieurs œuvres récentes» (Morel 1991: 29); dall'altro lato, dal fatto che l'oggetto di analisi è appunto una rappresentazione teatrale, cioè un'opera collettiva, come ha scritto S. Greenblatt (1988: 4-6). Pertanto essa trascende dalle mere intenzionalità dell'autore, e legittima le molteplici interpretazioni.

In questo senso l'obiettivo della lettura dell'evoluzione della tragedia francese degli anni 1630 è quello di arricchire le già numerose interpretazioni delle rappresentazioni drammatiche, apportando soluzioni che permettano di giustificarne a fortiori l'esistenza, tese in un certo senso à sauver les phénomènes. Si è cercato di mettere in luce il fatto che la creazione dei testi teatrali presi in considerazione non è estranea ai dibattiti teologico-filosofici coevi, e alle aspirazioni della politica culturale di Richelieu. Tali influenze sono implicite nelle scelte operate dal drammaturgo in fase inventio e dispositio, le quali si fanno così portatrici di senso, di un senso storico e politico.

\section{Bibliographie}

Agostino d'Ippona, Il Libero arbitrio, http://www.augustinus.it/italiano/libero_ arbitrio/index.htm

Ariosto L., 1990, Orlando furioso, Milan, Mondadori.

Aubignac F.H. (d'), [1657] 2001, La Pratique du Théâtre, Paris, Champion.

Bénichou P., 2011, Morales du grand siècle, Paris, Gallimard.

Bergson H., 1991, Essai sur les données immédiates de la conscience, Paris, PUF.

Blocker D., 1999, "Jean Chapelain et les lumières de Padoue. L'héritage italien dans le débat français sur l'utilité du théâtre (1585-1640)», Littératures classiques, 37, p. 97-116.

Bouchilloux H., 1997, «Descartes et Pascal: les raisons de l'action», in: L'action à l'âge classique, Philosophie, 53, p. 78-95.

Cassirer E., 1997, Descartes Corneille et Christine de Suède, Paris, Vrin, p. 1-37.

Caussin N., 1653, La Cour sainte, Paris, Bechet, t. II, p. 216-256.

Chapelain J., [1630] 2007, Opuscules critiques, Genève, Droz, p. 222-234.

Civardi J.-M., 2008, «Reflets et usages de l'Antiquité dans le théâtre de Mairet», Littératures classiques, 1/65, p. 65-81.

Corneille P., 1980, Euvres complètes, I, Paris, Gallimard, La Pléiade. 
Corneille P., 1987, Euvres complètes III, Paris, Gallimard, La Pléiade.

Corneille P., [1637] 2001, Le Cid, Paris, STFM.

Couton G., 1986, Richelieu et le théâtre, Lyon, Presses universitaires de Lyon.

Cyrano de Bergerac, [1654] 2005, La mort d'Agrippine, La Versanne, Encre marine.

Descartes R., [1637] 2000, Discours de la méthode, Paris, LGF.

Dotoli G., 1997, Temps des préfaces, Paris, Klincksieck.

Doubrovsky S., 1982, Corneille et la dialectique du héros, Paris, Gallimard.

Du Ryer P., [1637] 1986, Alcionée, in: J. Scherer, Théâtre du XVII siècle, Paris, Gallimard, La Pléiade, p. 85-142.

Duprat A., 2009, Vraisemblances. Poétiques et théories de la fiction, du Cinquecento à Jean Chapelain, Paris, Champion.

Forestier G., 1990, «Imitation parfaite et vraisemblance absolue. Réflexions sur un paradoxe classique», Poétique, 82, p. 187-202.

Forestier G., 1993, «De la modernité anti-classique au classicisme moderne. Le modèle théâtral (1628-1634)», Littératures classiques, 19, p. 87-128.

Forestier G., 1998, Corneille. Le sens d'une dramaturgie, Paris, Sedes.

Forestier G., 2004, Essai de génétique théâtrale. Corneille à l'œuvre, Genève, Droz.

Fumaroli M., 1996, Héros et orateurs, Genève, Droz.

Girard R., 2017, La violence et le sacré, Paris, Pluriel.

Giuseppe Flavio, 1974, La guerra giudaica, Milan, A. Mondadori, vol. I, p. 174-179.

Grimaldi N., 1988, Six études sur la volonté et la liberté chez Descartes, Paris, Vrin.

Hénin E., 2003, Ut pictura theatrum. Théâtre et peinture de la Renaissance italienne au classicisme français, Genève, Droz.

Hénin E., 2007, "Écriture et vision tragique dans la Cour sainte», in: S. Conte, Nicolas Caussin: rhétorique et spiritualité à l'époque de Louis XIII, Berlin, Lit Verlag coll. «Ars rhetorica», p. 103-120.

Jouhaud C., 1994, "L'écrivain et le ministre: Corneille et Richelieu», DixSeptième Siècle, 182, p. 135-142.

Louvat-Molozay B., 2008, «Frontières de la tragédie: La Silvanire, La Sophonisbe, La Sidonie», Littératures classiques, 65, p. 129-144.

Mairet J., 2004, Sophonisbe [1635], Théâtre complet I, Paris, Champion, p. 99-200.

Massip F., 2009, «Iconografia i espectacle: cap a una nova metodologia de la investigació teatral», in: F. Massip et al., Tarragona: espai festiu, espai teatral, Valls, Cossetània, p. 9-32.

Mazouer Ch., 2015, Théâtre et Christianisme. Études sur l'ancien théâtre français, Paris, Champion.

Merlin H., 1994, «Horace, l'équivoque et la dédicace», Dix-Septième Siècle, 182, p. 121-134.

Merlin H., 2009, «Réécriture cornélienne du crime: le cas d'Horace», Littératures classiques, 67, p. 101-114. 
Morel J., 1991, Agréables mensonges. Essais sur le théâtre français du XVII ${ }^{e}$ siècle, Paris, Klincksieck.

Platone, 2009, Repubblica, Milan, Bompiani.

Reina Li Crapi V., à paraître, «Rappresentazioni drammatiche (e aporie) della libertà in Shakespere (Hamlet), Calderón ( $L a$ vida es sueño) e Corneille (Le Cid)», in: A. Munari, E. Selmi, E. Zucchi (éds), Testi, tradizioni, attraversamenti: prospettive comparatistiche sulla drammaturgia europea tra Cinque e Settecento, atti del seminario dell'Università di Padova, 16-17 dicembre 2015, Padoue, Padova University Press.

Ricœur P., 1983, Temps et récit I, Paris, Seuil.

Scherer J., 1986, Théâtre du XVII ${ }^{e}$ siècle, II, Paris, Gallimard, La Pléiade.

Schmutz J., 2002, «Dal peccato dell'angelo alla libertà d'indifferenza. L'eredità angelologica dell'antropologia moderna», in: Fr.P.Adorno e L.Foisneau L'Efficacia della volontá nel XVII secolo, Roma, Edizioni di storia e letteratura, p. 57-79.

Tito Livio, 1997, Storie, volume IV, Turin, Utet, Libro XXX (12-15), p. 598-613.

Tristan L'Hermite, 2009, La Mariane [1636], in: Les Tragédies, Paris, Champion, p. 7-122.

Zanin E., 2014, Fins tragiques. Poétique et éthique du dénouement dans la tragédie pré-moderne en Italie, France et Espagne, Genève, Droz. 